\title{
Gendarmería Nacional Argentina y la gestión de los conflictos y la violencia en barrios informales del sur de la Ciudad de Buenos Aires
}

\section{Joaquín Zajac ${ }^{1}$}

\author{
1 Instituto de Investigaciones Gino Germani, Ciudad Autónoma de Buenos Aires, Argentina \\ (iD) http://orcid.org/oooo-ooo1-7836-8591 \\ Correo electrónico: joaquinz@gmail.com
}

Recibido:

30 de noviembre de 2019 Aceptado:

10 de octubre de 2020

doi: 10.34096/runa.v42i1.8731

\section{Resumen}

El objetivo del artículo es describir y analizar las intervenciones de Gendarmería Nacional Argentina (GNA) ante situaciones de conflictividad y violencia en barrios informales del sur de la Ciudad Autónoma de Buenos Aires entre 2016 y 2019, el marco de política pública y las representaciones institucionales que encuadran dichas prácticas. Analizo las tensiones y dificultades que conlleva para muchos efectivos de la Gendarmería intervenir ante demandas que desbordan funciones institucionalmente ponderadas como "propias". También, la forma en que dichas intervenciones iluminan una dimensión constructiva del "poder de policía", así como de una particular tecnología estatal de administración de los "márgenes", sus demandas y conflictos. El artículo se apoya en el análisis de fuentes documentales, y en un trabajo de campo etnográfico con efectivos de Gendarmería en el sur de CABA entre 2016 y 2017, y con habitantes de dichos barrios en 2019.

\section{Argentinian National Gendarmerie and management of conflicts and violence in informal neighborhoods of southern City of Buenos Aires}

\begin{abstract}
The aim of this article is to describe and analyze the interventions of the Argentine National Gendarmerie (GNA) in situations of conflict and violence in informal neighborhoods in southern Buenos Aires between 2016 and 2019, its public policy framework and the institutional representations involved in those practices. I analyze the tensions and difficulties that Gendarmerie has
\end{abstract}

Palabras clave

Gendarmería; Estado; Conflictividad; Violencias; Barrios informales 
to face addressing certain demands that exceed some institutionally weighted functions as "their own". Also, the way in which these interventions illuminate a constructive dimension of "police power", as well as the particular technology that the state uses for governing its "margins" and their demands and conflicts. The article is based on the analysis of documentary sources, and by an ethnographic fieldwork with members of the Gendarmerie in neighborhoods of the south of CABA between 2016 and 2017, and with inhabitants of these neighborhoods in 2019.

\section{Gendarmaria Nacional Argentina e a gestão de conflitos e violência em bairros informais no sul da Cidade de Buenos Aires}

\section{Resumo}

Palavras-chave

Gendarmaria; Estado; Conflito; Violência; Bairros informai
O objetivo do artigo é descrever e analisar as intervenções da Gendarmeria Nacional Argentina (GNA) em situações de conflito e violência em bairros informais no sul da Buenos Aires entre 2016 e 2019, o marco de políticas públicas e as representações institucionais que enquadram essas práticas. Analiso as tensões e dificuldades que acompanham a Gendarmaria, intervindo diante de demandas que excedem as funções ponderadas institucionalmente como "próprias". Além disso, a maneira pela qual essas intervenções iluminam uma dimensão construtiva do "poder policial", bem como uma tecnologia estatal específica para a administração de "margens", suas demandas e conflitos. O artigo é apoiado pela análise de fontes documentais e por um trabalho de campo etnográfico com membros da Gendarmeria em bairros no sul da CABA entre 2016 e 2017 e com os habitantes desses bairros em 2019.

\section{Introducción}

El presente artículo busca reflexionar sobre la presencia del Estado en zonas de alta vulnerabilidad social. Sobre cómo y porqué, demandas que podrían abordarse desde agencias especializadas se encuentran en la actualidad parcialmente recapturadas por dispositivos cuya finalidad no es la protección o ampliación de los derechos, sino lograr y mantener determinados niveles de seguridad. El objetivo general será describir y analizar las intervenciones de la Gendarmería Nacional Argentina ante situaciones de conflictividad y violencia en barrios informales del sur de la Ciudad Autónoma de Buenos Aires (CABA) entre 2016 y 2019, así como el marco de política pública y las representaciones de la propia fuerza que encuadran dichas prácticas. El trabajo dialoga con dos campos de estudios antropológicos.

En primer lugar, con quiénes desde la antropología han abordado a la Gendarmería y su quehacer desde distintos recortes problemáticos, aunque coincidiendo todos ellos en una preocupación común: las tensiones que ha provocado en la fuerza el crecimiento incesante-desde el regreso de la democracia- de su cantidad de efectivos, recursos, despliegue territorial y competencias. Según señalan estos distintos estudios, la Gendarmería es una fuerza 
de seguridad con doctrina, conducción, régimen disciplinario y, lo más importante, autopercepción militar (Frederic, 2018), inicialmente creada por el gobierno federal para apostarse en zonas rurales alejadas, poco pobladas, con escasa o nula presencia de otras burocracias (Escolar, 2017). Desde sus inicios, por la geografía en la que fue desplegada, se vería involucrada en graves conflictos con las comunidades de pueblos originarios, entre los que se destaca la llamada masacre de "Rincón Bomba" en Formosa en 1947 (Trinchero, 2009). Desde 1955, pasó a depender directamente del ejército, lo que reforzó su carácter militar y la llevó a involucrarse en actividades de represión ilegal, especialmente durante la última dictadura. Con el retorno democrático, ha impulsado una renovación de su imagen institucional, en la que se presenta como diferente tanto de los militares - por su pretendido mayor compromiso con a la democracia- como de los policías -por su supuesta honestidad, idoneidad y mayor "empatía" - (Escolar, 2017). Al mismo tiempo, el Estado nacional, imposibilitado por las leyes de Defensa (23.554) y Seguridad Interior (24.059) de emplear a las fuerzas armadas, y con policías desprestigiadas por numerosas acusaciones de corrupción y violencia institucional, se han valido crecientemente de esta "fuerza intermedia" para diversas misiones. Algunas, cuestiones estrictamente "policiales", ${ }^{1}$ como la represión de cortes de ruta en distintos puntos del país (Escolar, 2017). Otras, postuladas por la fuerza y algunos especialistas como "a medio camino" entre la seguridad y la defensa, como las "misiones humanitarias" internacionales y la asistencia en casos de catástrofe natural (Escolar, 2017), así como la prevención e investigación de los mencionados delitos del "crimen organizado transnacional" -narcotráfico, trata, terrorismo, etc.- (Renoldi, 2014a), tareas en las que otros países de la región han empleado fuerzas militares. Más recientemente, la fuerza ha ido sumando responsabilidades en materia de seguridad en zonas urbanas "conflictivas". Un contexto operativo para el cual no tenía preparación previa, y que la ha sumido en una traumática "policialización" (Frederic, 2018), lo que la obligó a producir sobre la marcha numerosas "adaptaciones" (Frederic, 2014), así como suscitado en su seno conflictos internos y desafíos a la cadena de mando (Frederic, 2015). Este trabajo busca aportar a este campo de estudios, en tanto permitirá poner en relación las intervenciones analizadas con esa historia institucional reciente, signada por la constante expansión y redefinición -no siempre armónica- de sus competencias y "funciones".

Un segundo campo de investigaciones con el que pretendo dialogar ha sido impulsado, fundamentalmente, por el equipo de Antropología Jurídica y Política de la Facultad de Filosofía y Letras de la UBA. En la matriz conceptual de estas investigaciones, se destaca fuertemente la noción de "poder de policía". El concepto, es definido por Tiscornia (1999), como un poder opaco por definición, impreciso en cuanto al dominio de sus objetos y ambiguo en cuanto a su relación con la legalidad, el derecho y la democracia. Un poder arbitrario, dirigido al "encauzamiento" de conductas públicas y privadas. Racionalidad política que trasciende a la institución policial, pero que se encarna principalmente en ella, en sus prácticas, reglamentos e ideología (Tiscornia, 1999, p. 81). Atraviesa a estos trabajos el propósito de relevar y sistematizar prácticas policiales, así como determinar las formas cambiantes en que articulan legalidad e ilegalidad, su "distribución diferencial" en distintos espacios (Pita y Pacecca, 2017, p. 43) y las formas en que se utilizan para "administrar" a diversos grupos sociales subalternos (Pita y Pacecca, 2017, pp. 46-47). En este sentido, en un trabajo de reciente publicación, Pita, Corbelle y Brescia (2019) realizan una evaluación de los efectos que han tenido las políticas de seguridad que analizo en este mismo artículo: los despliegues de policías y fuerzas de seguridad en barrios informales en el marco de los Cuerpos Policiales de
1. Esto ha generado numerosas críticas políticas y de los investigadores especializados, que ven en este proceso como una vía de "militarización" disimulada o encubierta (Salles Kobilanski, 2012; Hathazy, 2016). 
Prevención Barrial (CPPB) y, más recientemente, del Programa Barrios Seguros (PBS). Políticas que inicialmente se habían propuesto conciliar la "seguridad ciudadana" con la plena vigencia de los derechos humanos, pero que han ido alejándose, según las autoras, de esa formulación inicial. De acuerdo con la periodización con la que estructuran su análisis, a un primer período positivamente connotado (2011-2013) -con "presencia multiagencial" del Estado simultáneo al despliegue policial, así como un fuerte "gobierno político" de las fuerzas a partir de controles formales e informales- sobrevendrían luego etapas en las que el despliegue policial se fue "quedando solo", lo que generó, para las autoras, el "peor de los escenarios": una masiva presencia policial, sin controles ni acompañamiento de otras agencias estatales, cuyo principal efecto ha sido un marcado incremento en los niveles de "hostigamiento policial" (Pita et. al., 2019, pp. 58-63).

Mi intención es dialogar con esos hallazgos tanto desde un punto de vista tanto empírico como teórico. Empírico, al mostrar cómo la Gendarmería interviene no solo en una dimensión represiva, aumentando los niveles de hostigamiento, vigilancia y represión, y como lo han abordado otros estudios previos (Pita et. al., 2019; Zajac, 2020). También lo hace gestionando conflictos, a demanda de la propia población que, ante la presencia intermitente de otras agencias estatales de los territorios, no conoce y/o no encuentra disponible en muchos casos otro actor público al que recurrir. Asimismo, desde un punto de vista teórico, pretendo mostrar cómo el "poder de policía" poseería, además de su dimensión violenta y represiva, otra de "gobernanza constructiva" que procura la "pacificación" de los espacios sociales, en pos de "fabricar el orden social" (Neocleous, 2016).

El artículo se estructura en cuatro secciones. En la primera, detallo mi enfoque teórico y metodológico. En el segundo, los lineamientos de política pública que enmarcaron el despliegue y actividad de la Gendarmería al interior de las villas de la Capital, dando cuenta de las continuidades y rupturas entre las distintas etapas. En el tercero, muestro cómo los gendarmes intentan resolver la contradicción entre las tareas que deben realizar en los barrios informales y las funciones que son consideradas como propias y específicas. En el último, describo y analizo cómo las intervenciones de la fuerza constituyen una dimensión productiva del poder de policía, a la vez que configuran un tipo de presencia estatal en esos territorios, signada por la ambigüedad, la contingencia y las consideraciones securitarias.

\section{Perspectiva teórico-metodológica}

Siguiendo a Rosana Guber, considero a la etnografía como una concepción y práctica del conocimiento que busca comprender los fenómenos sociales desde la perspectiva de sus miembros (Guber, 2001, pp. 12-13), antes que como un método o conjunto de técnicas. En este sentido, sostengo que el enfoque etnográfico atravesó cada instancia de mi investigación, desde la construcción del objeto, hasta el proceso de análisis y escritura, pasando por el trabajo de campo y las técnicas de observación y registro. En cuanto a mi trabajo de campo, este inició en marzo de 2016, gracias a la autorización del comandante a cargo del "Operativo Cinturón Sur" (OCS) a quien pude contactar por intermedio de una exfuncionaria del Ministerio de Seguridad de la Nación (MSN) a quien conocí personalmente en una actividad política/académica. Desde los inicios, la obtención y sostenimiento del acceso al campo se basó en las relaciones de confianza, familiaridad y mutuo reconocimiento que mantuve con el jefe 
del OCS y el joven gendarme que oficiaba como su ayudante. Es decir, pude garantizar mi acceso al campo gracias al ejercicio sostenido de una práctica de la "reflexividad", que implica: 1. comprender que la valoración (positiva o negativa) de los nativos acerca de nuestro rol como investigadores es una condición inescindible, inevitable e ineludible de la investigación social; 2. tomar conciencia de que el acceso y permanencia en el campo no se garantizan de una vez y para siempre, sino en una prueba constante, en la que no existen guías $a$ priori para evitar "errores" ni una acumulación automática (Guber, 2014). Para el campo de estudios sobre el mundo policial, Renoldi (2014b) advierte muy acertadamente que es necesario tomar especiales recaudos, para evitar emular la variante de "investigación" que predomina en estos ámbitos: la averiguación del "secreto", su revelación, su denuncia. En este sentido, las jornadas de campo tenían una frecuencia semanal y resultaban de una negociación directa e informal con el ayudante del jefe, quien, luego de consultar con sus superiores, me asignaba el horario y lugar para la visita. A veces, era él quien me proponía instancias de observación. Otras ocasiones, yo formulaba algún pedido específico. Pero en todos los casos, el desafío metodológico principal era lograr un equilibrio entre mis inquietudes de conocimiento y el riesgo de que algunos de mis pedidos fueran "mal vistos" o pudieran confirmar a los gendarmes sospechas oscuras respecto de algún interés de mi parte en obtener información para perjudicarlos de alguna manera.

Esta práctica reflexiva y de priorización del acceso al campo por sobre todas las cosas implicó aceptar una elevada "rotación" de los lugares de observación, ya que según pude ir constatando, los distintos "jefes" de la fuerza se oponían a que observara sus divisiones de manera frecuente y sostenida. Dicha rotación me llevó a apostar entonces por una diversificación de contextos de actuación, antes que por la observación sistemática de un único contexto. Además, afectó de manera clara la elección de mis técnicas de observación y confección de "registros". Principalmente, me llevó a tomar la decisión de no grabar conversaciones ni tomar notas durante mis visitas. Esto permitía que, pese al desconocimiento y posible recelo, muchos gendarmes a quienes no llegaba nunca a conocer de manera íntima llegaran a confiar en mí, y así mantener conversaciones con un menor nivel de autocensura.

Posteriormente, en el curso de 2019, mantuve diversas entrevistas abiertas e hice "recorridas" de observación participante con habitantes de los mismos barrios de mi trabajo de 2016-2017 Se trataba de referentes comunitarios, sindicales y políticos, y un grupo de operadoras de un programa socioeducativo a los que pude contactar gracias a vínculos personales y/o laborales previos. Esta "revisita" al campo me permitió actualizar comprobar y complejizar algunas de los hallazgos obtenidos en mi estancia anterior.

En cuanto a los documentos que analizo en el artículo, estos son material de acceso libre y constituyen, por lo tanto, la cara más visible y pública de la política de seguridad. Contrastar dicha perspectiva pública con el punto de vista "nativo" de los gendarmes, de acceso restringido y recelosamente vigilado, podrá aportar información valiosa sobre la relación entre ambas "caras" de la acción del Estado.

Por último, en relación con mi posición teórica, adopto en este artículo una "antropología de los márgenes". Esta mirada propone distanciarse de la imagen del Estado como forma administrativa de organización política racionalizada que tiende a debilitarse o desarticularse en sus márgenes territoriales o sociales (Das y Poole, 2008, p. 19), para pensar a dichos márgenes como 
espacios en los que el Estado siempre está presente, experimentando y modificando las tecnologías que emplea para administrarlos y "pacificarlos". Y esto tanto por medio de la fuerza como de las "pedagogías de la conversión", que intentan transformar "sujetos rebeldes" en "sujetos legales" (Das y Poole, 2008, p. 24). "Pacificación" que, siguiendo a Mark Neocleous (2016), es el objetivo fundamental de los Estados modernos y sus políticas de "seguridad", desde sus inicios. Se trata de la búsqueda por obtener, no la destrucción de las poblaciones insumisas, sino su subordinación "pacífica". La construcción de un tiempo y un espacio de "paz" que le permita a la acumulación capitalista desarrollarse. Para esto, según este autor, los Estados se valen no solo de técnicas de violencia y destrucción de los grupos antagonistas, sino también de toda otra serie de esfuerzos de "gobernanza constructiva" de las poblaciones. Dualidad del poder estatal (represiva y constructiva) que intentaré reponer mediante los hallazgos de este artículo.

\section{De la policía barrial a los barrios seguros: continuidades y rup- turas en el modelo de policiamiento para barrios informales}

El primer antecedente de intervenciones de fuerzas de seguridad federales en barrios populares se remonta a noviembre de 2003. En el marco de una serie de casos resonantes de secuestros extorsivos, incluido el de Axel Blumberg (cuya muerte desencadenó masivas movilizaciones en reclamo de "seguridad"), el gobierno de Néstor Kirchner lanzó el "Plan de Protección Integral de Barrios" en tres localizaciones del conurbano bonaerense. La Gendarmería en particular fue desplegada en el complejo habitacional Ejército de los Andes ("Fuerte Apache"), estigmatizado por los medios de comunicación masivos como sinónimo de "peligrosidad" (Kessler, 2012). Entre 2010 y 2011 se produciría, no obstante, un salto cualitativo en este tipo de despliegues. Primero con el "Operativo Centinela" (6000 gendarmes en distintos puntos del conurbano bonaerense). Y luego, en julio de 2011, el OCS, que implicó el despliegue de 1250 gendarmes y 1250 prefectos en las comunas 4,7 y 8 del sur de CABA. ${ }^{2}$ Este operativo poseía una característica que lo diferenciaba de sus antecesores. La Gendarmería no actuaba en "concurrencia" con la policía local -la Policía Federal Argentina (PFA)-, sino que reemplazaba en casi todas sus competencias (con excepción de algunas administrativas y de auxilio a la justicia). El propio MSN fue creado pocos meses antes de estos operativos. Y en el primer número de su publicación Políticas de Seguridad presentaba al OCS como su anuncio más trascendente. En un juego de palabras que remitía a la canción del cantautor español Joan Manuel Serrat "El Sur también existe", el ministerio presentaba este desembarco de fuerzas de seguridad militarizadas como una política "progresista", "inclusiva". El sur de la CABA, se decía, era una zona "desprotegida", sin seguridad, de este modo recibiría una "compensación" por parte del Estado. La decisión de emplear a Gendarmería y Prefectura respondía a un "análisis" de la situación en el "sur porteño" que seguía "arrojando déficits" en el número de agentes policiales requeridos para "dar respuesta efectiva a la demanda de seguridad" (MSN, 2011, p. 3).

Sin embargo, a los efectos del trabajo, el hito más significativo se produjo entre octubre de 2011 y septiembre de 2012: la concepción y puesta en funcionamiento de los llamados "Cuerpos Policiales de Prevención Barrial" -CPPB(Basualdo, 2012). Se trataba de una nueva división de la PFA, creada para los barrios populares. Estos cuerpos estaban confirmados por voluntarios -oficiales y suboficiales en servicio-, quienes recibían un reentrenamiento específico antes de ser enviados a su nuevo destino. Los cuerpos de la PFA llegaron a 
las villas 21-24, la villa 31 y 31 bis, "Ciudad Oculta" y la Villa 20 (Pita et. al., 2019). La Gendarmería, por su parte, adoptaría esta modalidad en marzo de 2014, en las villas 1-11-14, Zavaleta y "Fátima".

Para analizar los lineamientos del CPPB me baso en la resolución 1034/12 que creó el cuerpo y en el segundo número de la ya mencionada publicación Políticas de Seguridad. En cuanto a la caracterización de los contextos de actuación, esta cambia sustancialmente en los CPPB, en comparación con el OCS. Se habla ahora no de la zona sur en general, sino de las "barriadas" o "barrios más humildes", a los que también se identifica como las "zonas de mayor conflictividad social", destacando que estas fueron desatendidas no solo en sus demandas de "seguridad" sino también frente a "las violencias". Los barrios pobres y sus "violencias" aparecen recortados como problema específico frente al de la "inseguridad" (Lorenc Valcarce, 2009; Galar, 2016), y que requiere intervenciones particulares.

Así, en la resolución de creación de la policía barrial aparecen como objetivos "prevenir la violencia", "contribuir a la resolución de conflictos" y la "convivencia pacífica". Esto incluye varios tipos de conflictividad: "conflictos comunitarios", "formas menores de conflictividad" (vinculadas a la "convivencia entre vecinos y en el espacio público"), "violencia familiar y de género", "violencia intrafamiliar o intervecinal". Para lograrlo, se menciona la necesidad de establecer "criterios y procedimientos de actuación policial tendientes a prevenir y disminuir la violencia en los conflictos comunitarios", específicamente, "acciones de mediación", "promover las vías de comunicación y desalentar el uso de armas de fuego".

Además, la resolución propone una serie de objetivos vinculados con el mejoramiento de las relaciones "Estado-comunidad": "canalizar las demandas y necesidades de los habitantes", "atender demandas de la comunidad vinculadas con trámites y denuncias", y "resolver situaciones que resulten de su competencia y/o realizando orientaciones y derivaciones efectivas". Los CPPB fueron presentados en simultáneo con el "Plan Nacional de Abordaje Integral" ("AHI'), una iniciativa que reunía en un mismo ámbito a agencias del Estado, organizaciones sociales y "redes comunitarias" (MSN, 2012). Los ministerios nacionales participaban, en cada barrio, en tareas de desarrollo social, salud, educación. A cada ministerio le tocaba "conducir" el plan en un barrio particular, y los CPPB eran considerados como "soporte" y acompañamiento (Basualdo, 2012). Con la posterior crisis y, luego, desarme del Plan AHÍ, esta intención de que las fuerzas de seguridad articularan su actividad con el de otras agencias públicas quedará, como veremos, en una declaración de intenciones vacía de contenido. Al mismo tiempo, se esperaba de estos cuerpos policiales el "desarrollo de contextos urbanos seguros", en el sentido de "garantizar la libre circulación de personas en el espacio público", así como de "erradicar la venta de estupefacientes" y "desactivar las redes delictivas". El ministerio veía a la posibilidad de circular por el espacio como "condición previa" para el ejercicio de los demás derechos. La estrategia para lograr esto era sostener una "presencia policial firme y confiable", diferente a la de la PFA. Esta distribuía a sus efectivos mediante "paradas", cuya ubicación, en muchos casos, se definía mediante un acuerdo económico informal entre vecinos/comerciantes y cada comisaría. La policía no "entraba" a las villas más que para hacer cumplir alguna orden judicial -allanamientos, detenciones, etc.(Frederic, 2014). Estos objetivos, en suma, se suponían -en la formulación de la política-como complementarios a los anteriores, pero en verdad responden a lógica incompatibles: los primeros promueven que las fuerzas de seguridad 
Es remarcable que este documento no fue publicado cuando el PBS se inició formalmente (abril de 2016 , en las villas 31 y 31 bis), sino al final de la gestión de gobierno, en 2019. De esta forma, más que una "presentación" o planificación de lo que iba a hacerse, es una "memoria"; un documento de "lecciones aprendidas" y de "resultados", que reflexiona sobre lo que las fuerzas efectivamente hicieron o buscaron hacer. se guíen siguiendo consideraciones no penales, de gestión de los conflictos y articulación con otras agencias públicas. El otro, que se apoyen en criterios policiales y penales, enfocándose en perseguir a quienes participaban de los mercados ilegales. La dificultad para conciliar ambas dimensiones será uno de los principales escollos que marcará, como veremos, las intervenciones de la Gendarmería.

Con la llegada de la Alianza Cambiemos al gobierno a fines de 2015 se produjo un evento de gran relevancia para el sistema de seguridad metropolitano: la creación de Policía de la Ciudad que unificaba en una misma institución a la Policía Metropolitana y a varias divisiones (principalmente, la Superintendencia de Seguridad Metropolitana), transferidas por el Estado nacional. Los CPPB dependientes de esta última fuerza también fueron transferidos, y prácticamente disueltos, ya que quedaron integrados casi únicamente por Gendarmería y Prefectura. Ambas fuerzas fueron paulatinamente retiradas del resto de la jurisdicción con el decreto de derogación del OCS (66/17), que redujo su presencia en CABA a algunos barrios populares en el marco del PBS.

En cuanto a los lineamientos de este programa, se observan a grandes rasgos algunas continuidades con respecto a los CPPB. Según se puede constatar en el "Manual" que el MSN publicó en el último trimestre de 2019, ${ }^{3}$ aunque el PBS propone intervención formada por cuatro "componentes" (el 0, de investigación judicial y encarcelamiento de grupos criminales, el 1; de despliegue policial de proximidad; el 2, de "fortalecimiento comunitario" a partir de actividades recreativas, educativas, culturales; y el 3, de intervención sobre el "medio físico" urbano, en línea con estrategias criminológicas de "prevención situacional del delito"), el despliegue policial continúa siendo el recurso más importante (MSN, 2019). Por otra parte, los barrios con "altos niveles de violencias" siguen definiéndose como los ámbitos específicos de intervención para los CPPB. Sin embargo, aparecen también modificaciones considerables. Por un lado, en cuanto al "lenguaje" y al marco conceptual utilizados. De una jerga liberal asociada a los derechos humanos, se pasa a un vocabulario técnico de la salud colectiva y la epidemiología que define a la violencia como una "enfermedad" colectiva con mayor "prevalencia" en territorios que concentran una proporción elevada de ciertos "factores de riesgo": ciertas "desventajas" o "privaciones relativas" de tipo urbano; características individuales (promedio de edad reducido, bajos ingresos, historial de abusos y violencia, etc.), interpersonales (familias "disfuncionales", grupos de pares "negativos", noviazgos violentos etc.), pero principalmente, "factores de riesgo comunitarios". La "falta de cohesión social/comunitaria" e "ineficacia de los "mecanismos de control social" serían los principales "agravantes" de la violencia que el PBS se propone "mitigar".

En este sentido, la reducción de las violencias aparece ahora, no como un objetivo más entre otros, sino como el propósito principal. Y esto no por una priorización genuina de la nueva gestión del MSN de la atención a las violencias por sí misma, sino porque controlar y reducir dichas violencias permitiría incidir en la "propensión criminógena" de los habitantes de estos barrios. Es decir, la mayor o menor habilidad individual para "autocontrolarse" ante la presencia de una "motivación" u "oportunidad" para cometer un delito, que sería causada o potenciada por los contextos interpersonales o comunitarios violentos (MSN, 2019, pp. 133-134).

Por lo tanto, a través de un despliegue policial "de proximidad" y "orientado a problemas", el PBS busca intervenir sobre el "medio instirriotucional": el conjunto de instituciones públicas que ejercen "control social formal", y que en 
estos barrios suele presentar "déficits de cobertura y/o calidad" que "erosionan la capacidad de prevenir, conjurar y mitigar las situaciones de violencia" (MSN, 2019, p.100). El PBS busca así "mejorar" esa cobertura para que los barrios dejen de ser contextos signados por un "cinismo moral/legal colectivo", en los que la ley se percibe como "existencialmente irrelevante", ya que

\begin{abstract}
la violencia se enseña, se aprende o contagia [...] inter-generacionalmente [...] a través de un set de [...] reglas informales de comportamiento, basadas en la creencia que en el espacio barrial no hay legitimidad alguna del sistema formal y las pautas culturales de la sociedad en general. Se genera así un mecanismo cultural de naturalización y perpetuación de conductas anti-sociales y violentas. (MSN, 2019, p. 100)
\end{abstract}

Para revertir esta situación, es necesaria una presencia policial "constante", "impactante", y, sobre todo, "flexible a las demandas y requisitos" cambiantes de los barrios. En suma, "flexibilidad", prioridad absoluta a la reducción de los niveles de "violencia" y captura plena de la orientación estratégica del despliegue hacia un sentido securitario, cuestiones que podrán apreciarse en los próximos apartados tanto en las intervenciones de Gendarmería, como en las representaciones que estos se formulan en torno a ellas.

\title{
De la frontera al barrio. Las resistencias de la Gendarmería a su rol de gestión de la conflictividad en barrios informales
}

Como ya he anticipado, Gendarmería ingresó a los CPPB en marzo de 2014. Desde entonces organizó a sus efectivos del OCS en dos tipos de estructuras diferenciadas. Por un lado, las "Unidades de Seguridad" (US) a cargo del territorio que correspondería a las comisarías de la PFA (34, 36 y 52). Por otro, las "Unidades de Prevención Barrial" (UPB), cada una de ellas en tres villas distintas: "Fátima", "1-11-14" y "Zavaleta". Al momento de hacer mi tesis, y según fuentes de la propia Gendarmería, de un total de 1762 efectivos en toda la CABA, cerca de 400, se encontraban destinados a las UPB. A este número se sumaba circunstancialmente una cantidad fluctuante de "agregados" (gendarmes que venían temporalmente desde otras jurisdicciones, por tres meses). En 2017, cuando mi trabajo de campo terminó, la jefatura me aseguró que con el lanzamiento del PBS en la villa 1-11-14, solo en esta ubicación el número de efectivos se había elevado en un número que oscilaba entre los 600 y 800 . Cada una de estas UPB contaba con jefatura propia, y todas ellas dependían a su vez de un "Destacamento de Prevención Barrial" (DESTAPREBA). A la fecha de mi investigación, dicha estructura administrativa no se había formalizado ni legalizado. La fuerza la mantenía como dependencia informal y "ad-hoc", fácilmente desarmable en caso de que poder ejecutivo nacional decidiera por fin efectivizar los numerosos rumores al respecto y levantar por completo el despliegue en CABA. Para la mayor parte de los gendarmes con los que hablé, aun los que estaban desde hacía más tiempo, estar en la ciudad era algo provisorio en sus carreras. No lo veían como un destino en el que quisieran quedarse, sino al contrario. Muchos, incluso, mantenían a sus familias viviendo en otros destinos, lo que les suponía tener que hacerse cargo de dos alquileres. Asimismo, ninguno de los gendarmes con los que conversé había recibido capacitación específica. Según el ayudante del jefe, la fuerza prefería no incorporar en los planes de estudio de oficiales y suboficiales, materias o incluso una especialidad en seguridad urbana, para evitar contribuir a que el despliegue en zonas urbanas se aceptara como permanente. 
Los registros burocráticos que producían en torno a las "incidencias" que debían atender cotidianamente en los barrios eran quizás la muestra más clara de cómo la fuerza interpretaba, clasificaba y valoraba negativamente su misión en estos contextos. Al principio del OCS, los "partes diarios" que elaboraba la fuerza cada jornada incluían una descripción pormenorizada de todos los "desplazamientos" de efectivos y patrullas. Pero desde 2013, con la centralización del servicio 911, Gendarmería solo guardaba registro de los desplazamientos "importantes", es decir, de aquellos que devenían en actuaciones judiciales. Lo prioritario para la fuerza era demostrar a las autoridades del Ministerio y a la opinión pública que Gendarmería no promovía "zonas liberadas" de determinados delitos (en 2016, particularmente del robo de vehículos, que se "iniciaba" en otras jurisdicciones, pero terminaba en la "zona de responsabilidad" de la fuerza). Estos "partes diarios" eran analizados y se utilizaban para planificar la dotación y distribución de los recursos operativos. El resto de las incidencias eran apuntadas en otro registro: las "actas de constancia", unos documentos breves cuyo único fin era resguardar a la fuerza de posibles reproches judiciales por negligencia. Si bien estas quedaban almacenadas en cada UPB, luego no eran tenidas en cuenta para la planificación. Esa omisión provocaba un "desfasaje" entre el resultado aparentemente exiguo de la actividad gendarme (medido en cantidad de hechos judicializados) y el gasto de recursos insumido (combustible, horas de trabajo), que solía ser motivo de cuestionamientos de las autoridades, lo que constituye una paradoja ya anticipada en los objetivos del CPPB: la judicialización de los conflictos no es un resultado deseable en términos de reducción de la conflictividad y la violencia, e incluso en muchos casos es lo contrario.

¿Por qué esta resistencia a la formalización, a la aceptación de este nuevo ámbito de actividad como parte de sus funciones estables? Los gendarmes definían el despliegue en barrios como algo ajeno, que desbordaba sus capacidades y, sobre todo, su experiencia previa. En especial, lo veían como contradictorio con el ámbito que creían "natural" a la institución: "las fronteras".

4. Hasta esta modificación, un operador de la PFA recibía y comunicaba las denuncias a cada comisaría. Esta, a su vez, daba o no aviso a Gendarmería, que, por último, decidía si "modulaba" el pedido a sus agentes. A partir del nuevo sistema, operadores civiles reciben los llamados y asignan las intervenciones directamente en base a proximidad, utilizando GPS (MSN, 2013). Además, toda la comunicación entre operador y agentes queda grabada, lo que compromete a los efectivos a acudir al lugar e informar el resultado de sus gestiones.

5. La GNA fue creada por la Ley 12.367 de 1938 , en la que no aparece ninguna mención a las fronteras. Recién con la sanción de las leyes 18.711 de 1970 y 19.349 de 1971 , quedó cristalizado dicho vínculo con la "zona de seguridad de fronteras", una "faja" de 50-100 km alrededor de fronteras terrestres, puentes y túneles internacionales. Existiría, entre la dinámica de "problemas" que encontraban en estas y la de los barrios, una diferencia cuantitativa. En las primeras, los problemas eran tan esporádicos que ellos mismos tenían que "salirlos a buscar". Mientras que en los barrios los "problemas" son tantos que "vienen solos". Al estar localizados en garitas "adentro" de los barrios, los gendarmes pueden recibir más fácilmente denuncias y pedidos de ayuda. Además, los efectivos destacaban como factor fundamental cambios en la operatoria de los llamados de emergencia (línea 911) ${ }^{4}$ que habían multiplicado su volumen hasta convertirlos en el aspecto que más tiempo les insumía diariamente.

Aparte de esta diferencia cuantitativa, habría otros contrastes "cualitativos". Las tareas que realizan a diario en los asentamientos eran percibidas por ellos como contrarias a su vocación, historia institucional y funciones definidas por ley. ${ }^{5}$ Como ya he anticipado, para los gendarmes, la fuerza se caracterizaría -además de la seguridad en fronteras- por investigar "delitos complejos", "del fuero federal". Delitos que, por el carácter transnacional de las organizaciones criminales que los llevan a cabo, afectarían a la "soberanía nacional" y, por lo tanto, serían acordes para una fuerza militarizada. Según los propios gendarmes de distinto rango con los que conversé en mi trabajo, la mayoría de las "incidencias" o pedidos de intervención en los cuales eran cotidianamente convocados ni siquiera eran delitos "ordinarios": en la mayoría de los casos, no devenían siquiera en procesos judiciales. 
Es decir, la resistencia a formalizar su presencia en los barrios, a someterse a capacitaciones específicas y a otorgar a los conflictos no judicializables la misma jerarquía que a los "delitos federales" (como el tráfico de drogas) puede entenderse en el marco de una resistencia de la Gendarmería a la "policialización" a la que se ve expuesta la fuerza a medida que se extiende en el tiempo su presencia en barrios urbanos (Frederic, 2018). Durante la revisita al campo en el año 2019, he podido comprobar como, si bien el rol de Gendarmería en la intervención ante estos conflictos se mantenía vigente en una cantidad significativa de situaciones, en muchos otros casos, había efectivos que se negaban a intervenir alegando que "no están en el barrio para ocuparse de eso". Negativa que facilitaba la derogación del Decreto 864/11 del OCS en enero de 2017, ya que ese marco legal los forzaba legalmente a atender todas aquellas situaciones que corresponderían por defecto a la PFA.

En suma, se puede ver cómo objetivos que parecían complementarios en la formulación de los CPPB no fueron asimilados de manera plena y genuina por las fuerzas que debían llevarlos a cabo, en especial, para el caso de Gendarmería, por encontrarse en conflicto con su carácter militar y la concepción que mantienen sobre su rol y sus funciones. Esta negativa a aceptar estas nuevas competencias y obligaciones, de intentar mantenerlas como provisorias, afectaba -además de los aspectos que he señalado en este apartado- el carácter mismo de las intervenciones, tal y como demuestro en la próxima sección.

\section{Conteniendo desbordes. Intervenciones de Gendarmería ante conflictos y violencias, sus efectos y sus límites ${ }^{6}$}

En este apartado se analiza solo una selección breve de situaciones observadas durante mi trabajo de campo. Se trata de hechos de violencias y conflictividad en los que intervienen los gendarmes empleando diversas tácticas policiales que constituyen una dimensión no violenta ni represiva del poder policial (Tiscornia, 1999). Empleo para agruparlos la propia clasificación nativa: "bajar gatos del árbol", "apagar incendios", y "hacer de psicólogo".

En cuanto al primer grupo, eran eventos en los que no había violencia interpersonal, infractores ni víctimas. Casos que se vinculaban a problemas diversos e intrincados, como, por ejemplo, cuestiones de infraestructura urbana o de los servicios públicos (recolección de residuos, electricidad, agua, etc.). El tipo de respuesta que ensaya Gendarmería en estas situaciones era atender sus externalidades más urgentes e inmediatas dejando sin solución los problemas de "fondo" que los originaban. En la escena, el fuego del auto en llamas es apagado, pero la carrocería abandonada seguirá allí, sin recoger, como inicialmente reclamaban los vecinos.

Era mi primera de campo jornada a la noche. Me habían avisado que iba a estar "complicada": había una fiesta religiosa de la comunidad boliviana en la capilla del barrio, con "riesgo de disturbios", por lo que tendrían a la mayor parte de los efectivos dedicados a vigilar el evento [...] Me recibieron en la parte de atrás de la parroquia dos suboficiales y un oficial. Empezamos a hablar un poco de mi trabajo, la típica charla explicando qué estaba haciendo y para qué. Entonces comenzaron a hablar ellos, discutiendo con indignación contra la idea de que "el Estado no está presente" en las villas:

- ¿Vos viste a tu alrededor? Tienen de todo. Salitas, escuelas, canchitas de fútbol. Hay mucho más acá que de dónde venimos, que hay repoco y está todo lejos - dijo el oficial de turno.
6. He decidido excluir de los análisis de este artículo un tipo de violencias en particular: las violencias de género. Tal decisión ha sido premeditada, ya que considero que, por la complejidad particular que encarnan esas situaciones, es necesario para abordarlas un análisis específico, que será el objeto de futuros escritos. 
-Viven acá porque quieren. Están cerca de todo, tienen todo gratis. Y si se corta cinco minutos la luz, o el agua, cortan la calle, prenden fuego gomas, $y$ tenemos que venir nosotros, llamar a la empresa -agregó uno de los dos suboficiales [...]. Al rato de estar en el puesto conversando, ocurrió la primera "incidencia" de la noche. Enfrente, un auto abandonado empezó a arder. Los gendarmes llamaron al camión de bomberos de la PFA. Uno de los suboficiales se acercó a charlar con vecinos que se habían acercado y nos dijo cuál era la teoría del barrio.

-Dicen que fue el que lo tenía en la puerta de su casa. Lo prendió fuego a ver si lograba que se lo lleven. Pero como el auto no tiene pedido de secuestro, no sirve. Acá podés estar años esperando que vengan a llevarse un auto abandonado. Tenemos un montón de reclamos así, nosotros los anotamos e insistimos, pero si la municipalidad no viene, no podemos hacer nada. La gente tiene miedo que se meta alguien a vivir adentro. A los 15020 minutos, el camión llegó y apagó el fuego, pero luego, dejó la chatarra chamuscada en el lugar. (Registro de campo, UPB 1-11-14, CABA, agosto de 2016).

Pero si en el caso anterior la expresión "apagar el incendio" se refiere a una intervención literal, en otros aparece como concepto nativo para clasificar un tipo particular de situaciones:

Estaba en la base conversando con el alférez K., cuando "entró" un llamado del 911: una "pelea vecinal". La descripción del hecho era breve, confusa. Un hombre joven estaba insultando a los gritos y acusando de "transa" a otra vecina. Me dejaron acudir en un patrullero blindado aclarándome que, si se llegaba a "complicar", debería esperar adentro. Al llegar pude ver entre la multitud al joven (25-30 años), muy delgado, con el torso desnudo, shorts gastados, sandalias y medias. K. pidió refuerzos por la radio. A los 10 minutos llegaron cinco patrulleros con 15-20 gendarmes más, con las balizas encendidas. El brillo e intermitencia enceguecían. Se escuchaban gritos constantes. En un momento, se hizo relativo silencio, y K. se acercó a conversar con los gendarmes que habían llegado antes.

- La señora dice que le pegó - me dijo-. Si hay agresiones, tengo que detenerlo. Nos está gritando aparte, eso es resistencia a la autoridad ¡Vení! -le gritó al joven. K. volvió donde se encontraba el joven. Pude ver cómo discutían y cómo K. empujaba con fuerza la cabeza del joven por debajo del marco de la puerta de un patrullero. Dos suboficiales, uno de los cuales portaba una escopeta, impedían el paso a un señor mayor que se quería acercar, protestando, alegando que el joven no había hecho nada. K. se quedó un rato charlando con el suboficial de la escopeta, que tenía algunas manchas de sangre en el brazo.

- Me dijo que estaba todo tranquilo, pero después se pudrió, se equivocaron. No dimensionaron el nivel de bardo que había - dijo mientras volvíamos a la base.

Una vez allí, los gendarmes se pusieron a redactar "actas de constancia". K. me llamó a un costado de la base, donde se encontraba el joven, más calmado.

-El muchacho acá es sociólogo, contale lo que me estabas diciendo.

-No bueno, con todo respeto... yo había ido a cortar el mambo que tenía, a comprar un poco de droga. Le pedí a la vendedora que me fiara, ella me conoce, y... No pudo continuar con el relato. Aparecieron dos mujeres mayores (40-50) y una más joven (20-25), parientes del joven.

- ¿Estás bien? ¿Qué te pasó? — preguntó la más joven, exaltada.

-No, nada, tuve problemas con P.

-¿Qué te hizo la transa esa? La voy a buscar y la voy a moler a palos a esa, atrevida. ¿Te lastimó?

-Disculpen -interrumpió K.-. Si la señora, no hace falta que la vayan a buscar. Vamos a la comisaría, y hacen la denuncia. Me parece la mejor solución. - Las mujeres hicieron silencio y se miraron. 
—No, está bien —dijo la mayor-. Vení, vamos a casa mejor —dijeron al joven-. Te das una ducha. ¿Se puede ir?

-Sí, claro.

Según K., al no haber lesiones o denuncia, no podía actuar de oficio, tenía que dejarlo ir. La "transa" era un problema para inteligencia, pero eso lleva tiempo. "Más si nadie denuncia”. (observación de campo, UPB Fátima, CABA, diciembre 2016)

En la escena puede apreciarse el carácter contingente y policial de las respuestas de la Gendarmería ante este tipo "incendios" de violencia intervecinal. De una parte, la nula multiagencialidad con otras agencias del Estado: aunque el joven denunciado se encuentra de manera notoria en un estado psicológico alterado debido al consumo problemático de sustancias, los efectivos no consideran en ningún momento la posibilidad de dar intervención al sistema de salud, tal y como establece incluso el protocolo del MSN respectivo. ${ }^{7}$ Por otro lado, se hace patente la impotencia de la fuerza para lograr encuadrar judicialmente esas situaciones, hecho que les produce una gran frustración y enojo con la población de los barrios, y que da cuenta de cómo en ellos la Gendarmería tiene legitimidad como recurso para la gestión de los conflictos, que no implica una legitimidad concomitante del sistema penal en su conjunto.

Adicionalmente, cabe remarcar cómo, si bien en la escena se produce una "detención" (arresto y posterior traslado de un individuo), no puede decirse que esta posea el mismo carácter y consecuencias que las que Tiscornia (1999) analiza en sus trabajos sobre poder policial (detenciones por averiguación de identidad, "razzias", procedimientos contravencionales, etc.). En este caso, el destino de dichas detenciones no es la comisaría o la cárcel, sino la "base" de la "UPB" de Gendarmería. Se trata de una "detención "informal", sin consecuencias judiciales, y de la cual no queda otro registro que un "acta de constancia". Y que si bien comporta ciertas prácticas violentas o punitivas (en la escena, algunos empujones y una reducción brusca) poseen, no obstante, un sentido productivo/constructivo antes que de reglamentación represiva de las conductas: "separar" a las partes, "apagar incendios", "contener" los desbordes.

Por último, estaban aquellos casos que la fuerza clasificaba como "hacer de psicólogo", y que involucraban, generalmente, a integrantes de un mismo grupo familiar:

Volvíamos a la base de la UPB después de un patrullaje, cuando se acercó corriendo y llorando una chica joven (15-20 años aproximadamente).

_ ¡Me sacaron a mi bebé!¡Ayúdenme!

Un grupo de cinco gendarmes, incluido el primer alférez $\mathrm{W}^{8}{ }^{8}$ empezaron a prepararse - ¿Querés venir? - me preguntó W.

Empezamos a caminar, tres gendarmes y yo, con la chica que había denunciado el hecho adelante.

Los suboficiales caminaban adelante con la chica, yo con W. un poco más atrás. Nos adentrábamos por una calle de cemento sin asfaltar. A las pocas cuadras, llegamos a una casa de ladrillo a la vista, en construcción. Un hombre mayor vestido con ropa de trabajo bajó del primer piso y abrió la puerta.

-Buenos días. Venimos por el asunto del bebé. Le pregunto ahora para evitarnos inconvenientes ¿Van a colaborar todos? - preguntó W.

- Sí, subamos.

Además del living-cocina, la casa tenía dos cuartos pequeños. En uno aguardaban en silencio dos chicos que parecían hermanos (14-16 y 20-25). Ambos vestían ropa deportiva y gorras. Según pude reconstruir, el bebé no había sido "robado" por un desconocido, sino por su ex (el hermano mayor), a pedido de la exsuegra, que
7. Según la Resolución 506/13 del MSN, se espera de los efectivos policiales que den intervención a un equipo de salud como estrategia principal ante este tipo de situaciones de urgencia.
8. Es llamativo como incluso en situaciones en las que una presencia o conducción femenina podía ser positiva (por tratarse de denunciantes mujeres, que probablemente se sentirían más cómodas tratando con una gendarme de su mismo sexo), no he podido observar intervenciones conducidas o acompañadas por gendarmes femeninas. No poseo datos que me permitan explicar esta situación, aunque es notable que no pude observar durante mi trabajo de campo un gran número de muchas destinadas a las UPB, y que las que conocí, estaban predominantemente abocadas a tareas de menor riesgo, como tareas administrativas, periciales y solo en algunos pocos casos, de "prevención" (como patrullajes o controles). 
quería llevarlo al hospital. La exsuegra (40-50 años), mecía al bebé, intentando calmarlo. El niño observaba aterrado la escena. No lloraba, pero tosía y moqueaba constantemente.

-El chiquito está enfermo, necesita ver a un médico. Ella está todo el día de aquí para allá, y se lo lleva con ella. Yo la quiero como la hija que no tuve. Tiene sus diferencias con mi hijo, yo lo entiendo, pero tiene que entender que el bebé no se siente bien, necesita cuidados - dijo la mujer, con voz calma y un tono maternal. -iY a vos que te importa si me llevo al nene, no me molesta! El otro que se la pasa basureándome entra a mi casa sin permiso, no me quiere devolver la llave, me saca al nene -el muchacho en el cuarto de al lado sonrió, e intentó responder las acusaciones. Pero la chica lo interrumpió.

_-iCallate vos! Y al médico no voy, ya sé lo que me va a decir, se tiene que nebulizar, y yo no tengo nebulizador.

-Tranquila. ¿No ves? Yo entiendo que tengas diferencias con él. Pero tu exsuegra te quiere ayudar, te quiere como a su hija, más allá de que vos por ahí a él no lo quieras más -la interrumpió W.-. ¿Por qué no te dejás ayudar?

La tensión parecía aminorar. La chica aceptó que la exsuegra llevara al nene al hospital. El hombre de pelo entrecano bajó a abrirnos y nos fuimos.

- ¿Cómo hacés para intervenir en situaciones como estas, en qué te apoyás? -le pregunté a W.

$-Y . .$. en el saber que uno tiene de ser padre, de tener familia. El chico siempre es una bendición, pero a veces son muy jóvenes estas chicas yno saben cómo ocuparse. (observación de campo, UPB Fátima, CABA, abril 2016)

Inicialmente el conflicto descripto en la escena parece tratarse de un delito grave: el robo de un bebé. Sin embargo, a los pocos minutos descubrimos que el asunto guardaba otra complejidad. Si bien la sustracción del niño efectivamente había tenido lugar, esta había sido efectuada por una persona conocida de la denunciante, con quien esta mantenía un vínculo personal, afectivo, muy cercano al parentesco. Un conflicto de índole familiar, como los que, muy probablemente, ocurren diariamente en otros puntos de la ciudad, pero que en barrios informales presentan condiciones específicas que los agravan y/o complejizan: falta de acceso a instituciones de cuidado de niños/as, hacinamiento habitacional, precariedad laboral, acceso a la salud exclusivamente por medio del sistema público (con sus conocidas demoras), dificultades en el acceso a la justicia y las burocracias estatales en general, etcétera.

Ante este tipo de conflictos, la fuerza procedía a realizar lo que consideraba una "intermediación". Una práctica policial que involucraba a los gendarmes en tramas vecinales/comunitarias y de parentesco, en sus disputas y sus relaciones de poder. Y en la que, ante el desconocimiento de protocolos y técnicas específicas para lidiar con dichas situaciones, muchos efectivos se veían llevados a implicarse emocionalmente como herramienta de intervención. Así como también, a poner en juego su propia concepción particular sobre las relaciones de pareja, la familia, la crianza de los hijos, etcétera. Involucramiento emocional y moral que implicaba, como asegura Frederic (2015), niveles intolerables de angustia y estrés, desde los primeros momentos del OCS. Muchos gendarmes, además, agregaban que para ellos, en estas situaciones, "el uniforme" no los ayudaba, ya que generaba en las personas vulnerables que requerían su ayuda temor o desconfianza. Y que lo que hacía falta era que profesionales de las ciencias humanísticas (psicólogos, trabajadores sociales, etc.), con la "sensibilidad" y los conocimientos adecuados, vinieran a los barrios y se ocuparan de intervenir ellos ante esos conflictos. La articulación con otras agencias especializadas aparecía en esos casos, no como una realidad institucional efectiva, sino como una expresión de deseo formulada en abstracto por los gendarmes. 


\section{Consideraciones finales}

En este artículo me he propuesto describir y analizar prácticas de la Gendarmería ante demandas y formas de conflictividad en villas del sur de CABA, así como las concepciones de política pública y representaciones de la propia fuerza que encuadran dichas prácticas. En un primer apartado he demostrado que objetivos que en la formulación de política pública se habían pensado como complementarios resultaban en verdad contradictorios entre sí. Así, una misma situación conflictiva es factible de ser interpretada e intervenida como un delito que hay que reprimir, un conflicto que hay que "intermediar", o una situación social compleja que requiere de la respuesta multiagencial del Estado. Por otra parte, "combatir el crimen organizado", "prevenir la violencia y mediar los conflictos", y "canalizar demandas" suponen esquemas de percepción, clasificación, saberes, habilidades y destrezas muy distintos. Es poco probable que efectivos de una fuerza de seguridad con características militares, con formación y experiencia en otro tipo muy distinto de ambientes operativos y tareas, logren asimilar todas ellas y resolver luego adecuadamente en cada caso qué medida corresponde aplicar. Posteriormente, las autoridades del MSN han recogido e intentado resolver dicha contradicción reformulando el marco hacia una orientación mucho más securitaria. La reducción de violencias se configura como objetivo principal, no por su valor intrínseco sino porque barrios menos violentos ayudarían a reducir la "propensión criminógena" de quienes habitan en ellos. La respuesta "contenedora" del Estado ante las "violencias" y los conflictos es una necesidad securitaria antes que una política de dignificación y expansión de los derechos de estas poblaciones.

En el segundo apartado muestro cómo la incorporación de estos lineamientos ambiguos de política pública ha afectado a la propia Gendarmería. El carácter de fuerza intermedia y militarizada, junto con la misión institucional que la fuerza asumió como proyecto desde 1990 (el combate contra las "nuevas amenazas", especialmente el narcotráfico), hace que muchas situaciones "no judicializables" sean percibidas por los gendarmes como problemas de jerarquía e importancia menores, que "rebajan" su prestigio institucional. Ese rechazo se refleja de diversas formas: en el tipo de registro burocrático degradado que se produce en torno a ellas, en la casi nula importancia que tienen para la planificación operativa, en la escasa formación específica y en las intervenciones en sí.

Fallando permanentemente en sus intentos por encuadrar judicialmente los conflictos en los que intervienen cotidianamente (ya porque se trata de hechos que no se prestan a su resolución mediante la vía judicial, o por la persistente ilegitimidad de la burocracia judicial en los barrios), las salidas empleadas por los gendarmes son unas tácticas policiales que apenas logran "contener" las emergencias, evitar los "desbordes" más violentos. La derivación a otras agencias del Estado rara vez ocurre, ya porque las agencias se han retirado de los territorios, porque los gendarmes las desconocen o porque estas están desbordadas, con una dotación de recursos insuficiente para atender los déficits de acceso a derechos que constituyen el verdadero sustrato de muchos de los conflictos que aquejan a estos barrios.

En suma, he mostrado cómo el despliegue de gendarmes en los barrios informales constituye algo más que una militarización de la seguridad con consecuencias estrictamente represivas Existe una variante "productiva" del poder de policía que complementa a su dimensión represiva de encauzamiento de las conductas (Tiscornia, 1999). Ambas dimensiones se encuentran en definitiva orientadas al mismo fin, la administración de poblaciones. Y configuran un 
tipo de actividad del Estado en estos contextos a medio camino entre la represión policial y la "protección" que correspondería a las agencias de "política social". Una "tecnología de gobierno de los márgenes" (Das y Poole, 2008) que evidencia la improductividad de pensar la presencia estatal como nula o débil en estos contextos. De lo que se trata es de investigar de qué forma, con qué división de competencias y funciones, las burocracias estatales se articulan en distintas estrategias de gobierno. Entender al "ejercicio de la violencia" y la "gobernanza constructiva" como momentos de un mismo proceso de "pacificación" permite superar ciertas "falsas dicotomías" en la comprensión de la naturaleza del poder del Estado (Neocleous, 2016, p. 14).

Para finalizar, una última consecuencia que considero se deduce de las indagaciones de este artículo. Considero vital que los debates políticos sobre fuerzas policiales y sus roles no pierdan de vista la distinción entre la constatación empírica de lo que ocurre, la evaluación crítica de cómo lo hace, y el posicionamiento ético y político sobre si esto que ocurre es o no deseable. En este sentido, el hecho de que fuerzas policiales crezcan no solo en número y alcance territorial, sino en funciones y competencias; que sean utilizadas no solo para vigilar, reprimir, o capturar, sino también para suplir déficits de otras agencias públicas, atendiendo demandas que desbordan su historia, proyectos institucionales, capacidades, etc., es, efectivamente, algo que está sucediendo y, posiblemente, con cada vez más frecuencia. Pero no es, en primer lugar-como permite ver el artículo-, algo que pueda ser evaluado positivamente, ni tampoco algo deseable. Considero que las fuerzas de seguridad pueden tener un rol, una función en el marco de una política pública multiagencial de los cuidados (evitar formas autogestionadas de resolución de los conflictos, contener desbordes, hacer cesar la violencia, garantizar la seguridad de los operadores estatales, etc.), pero nunca constituir el núcleo de dicha política. No se trata de un argumento puramente técnico. En una coyuntura de crecimiento de movimientos, líderes, discursos y subjetividades autoritarias en toda la región, la dependencia excesiva del poder político respecto de las fuerzas de seguridad constituye un serio riesgo para la democracia que no puede ser soslayado.

\section{Financiamiento:}

Este documento ha sido posible gracias a una Beca Interna Doctoral del Consejo de Investigaciones Científicas y Técnicas (CONICET).

\section{Agradecimientos:}

Agradezco a mis directores de tesis, Silvia Guemureman y José Garriga Zucal, por su acompañamiento y generosidad.

\section{Biografía}

Licenciado y profesor en Sociología. Magíster en Antropología Social. Doctorando en Ciencias Sociales. Integrante del Observatorio de Adolescentes y Jóvenes (IIGG-UBA), y del Núcleo de Estudios sobre Violencia y Muerte (IDAES-UNSAM). Profesor adjunto de Metodología de la Investigación (USAL). 


\section{Q Referencias bibliográficas}

》 Basualdo, G. (diciembre, 2012). Nuevas intervenciones territoriales en políticas de seguridad: una reflexión sobre el Cuerpo Policial de Prevención Barrial. Ponencia presentada en las VII Jornadas de Sociología, Universidad Nacional de la Plata, La Plata, Argentina.

"Das, V. y Poole, D. (2008). El Estado y sus márgenes. Etnografías comparadas. Cuadernos de Antropología Social, 27, 19-52.

»Escolar, D. (2017). Gendarmería. Los límites de la obediencia. Buenos Aires: SB.

» Frederic, S. (2014). Modos de dar seguridad, adaptación y obediencia en el escenario de re-despliegue territorial de la Gendarmería Nacional Argentina. Estudios, 32, 219-241.

》 Frederic, S. (2015). ¿Militares, asalariados o trabajadores? Moral y emoción en un conflicto gremial de la Gendarmería Nacional Argentina. Dilemas, 8(3), 529-557.

"Frederic, S. (2018). La politización del trabajo policial en Buenos Aires. Gendarmes y policías locales frente al policiamiento de proximidad. Trabajo y Sociedad, 31, 33-51.

»Galar, S. (2016). La agenda de la seguridad en revisión: casos conmocionantes, temas y problemas públicos en el actual período democrático argentino (1983-2016). Sociohistórica, 37, 1-13.

》Guber, R. (Comp.) (2014). Introducción. En Prácticas etnográficas. Ejercicios de reflexividad de antropólogas de campo (pp. 13-41). Buenos Aires: Miño y Dávila.

" Hathazy, P. (2016). La (re)militarización policial en la Argentina post-crisis: entre intereses organizacionales e instrumentalización política en los campos policiales. Dilemas, 9(1), 181-213.

» Kessler, G. (2012). Las consecuencias de la estigmatización territorial. Reflexiones a partir de un caso particular. Espacios en Blanco, 22, 165-197.

»Lorenc Valcarce, F. (2009). Orden, derechos y delitos La inseguridad en la agenda pública argentina. El Príncipe, 29 (2), 83-107.

» Ministerio de Seguridad de la Nación (2011). El Estado responde a las necesidades de seguridad. Políticas de Seguridad, 1, 3-6.

» Ministerio de Seguridad de la Nación (2012). El Estado nacional presente en los barrios vulnerables, Políticas de Seguridad, 3, 3-6.

» Ministerio de Seguridad de la Nación (2013). Nuevo Centro de Comando y Control de la Policía Federal Argentina, Políticas de Seguridad, 4, 3-4.

» Ministerio de Seguridad de la Nación (2019). Manual del Programa Barrios Seguros. Buenos Aires: Ministerio de Seguridad de la Nación Argentina.

» Neocleous, M. (2016). La lógica de la pacificación: guerra-policía-acumulación. Athenea Digital, 16(1), 9-22.

»Pita, M. V. y Pacecca, M. I. (2017). Territorios de control policial. Gestión de ilegalismos en la Ciudad de Buenos Aires. Buenos Aires: Facultad de Filosofía y Letras, UBA.

》 Pita, M. V., Corbelle, F. y Brescia, F. (2019). Las formas de la violencia policial: anatomía y gramática del poder policial en los barrios populares de la Ciudad de Buenos Aires. Recuperado de https://bit.ly/2WsldWz 
»Renoldi, B. (2014a). El secreto, el informante y la información: indagaciones reflexivas sobre la etnografía y la investigación policial. En R. Guber (Comp.), Prácticas etnográficas. Ejercicios de reflexividad de antropólogas de campo (pp. 113-138). Buenos Aires: Miño y Dávila.

»Renoldi, B. (2014b). Carne de carátula. Experiencias etnográficas de investigación, juzgamiento y narcotráfico. Buenos Aires: Ediciones Al Margen.

» Salles Kobilansky, F. (2012). ¿Militarización sin militares? Los gendarmes en las calles argentinas durante los gobiernos kirchneristas (2003-2012). Urvio, 12, 13-24.

"Tiscornia, S. (1999). Entre el imperio del “Estado de policía” y los límites del derecho. Seguridad ciudadana y policía en Argentina. Nueva Sociedad, 191, 78-89.

" Trinchero, H. (2009). Las masacres del olvido. Napalpí y Rincón Bomba en la genealogía del genocidio y el racismo de estado en la Argentina. Runa, 30 (1), 45-60.

»Zajac, J. (2020). Presencia de Gendarmería Nacional Argentina y sus efectos para las y los jóvenes de barrios informales de Buenos Aires. Erbio, 8(15), 161-190. 